\title{
Neutrosophic Chromatic Number Based on Connectedness
}

\author{
Henry Garrett \\ Independent Researcher \\ DrHenryGarrett@gmail.com \\ Twitter's ID: @DrHenryGarrett | CDrHenryGarrett.wordpress.com
}

\begin{abstract}
New setting is introduced to study chromatic number. vital chromatic number and $\mathrm{n}$-vital chromatic number are proposed in this way, some results are obtained. Classes of neutrosophic graphs are used to obtains these numbers and the representatives of the colors. Using colors to assign to the vertices of neutrosophic graphs is applied. Some questions and problems are posed concerning ways to do further studies on this topic. Using vital edge from connectedness to define the relation amid vertices which implies having different colors amid them and as consequences, choosing one vertex as a representative of each color to use them in a set of representatives and finally, using neutrosophic cardinality of this set to compute vital chromatic number. This specific relation amid edges is necessary to compute both vital chromatic number concerning the number of representative in the set of representatives and n-vital chromatic number concerning neutrosophic cardinality of set of representatives. If two vertices have no vital edge, then they can be assigned to same color even they've common edge. Basic familiarities with neutrosophic graph theory and graph theory are proposed for this article.
\end{abstract}

Keywords: Neutrosophic Connctedness, Neutrosophic Graphs, Chromatic Number AMS Subject Classification: 05C17, 05C22, 05E45

\section{Background}

Fuzzy set in Ref. [15], neutrosophic set in Ref. [2], related definitions of other sets in Refs. [2,13,14], graphs and new notions on them in Refs. [5-11], neutrosophic graphs in Ref. [3], studies on neutrosophic graphs in Ref. [1], relevant definitions of other graphs based on fuzzy graphs in Ref. [12], related definitions of other graphs based on neutrosophic graphs in Ref. [4], are proposed.

In this section, I use two subsections to illustrate a perspective about the background of this study.

\subsection{Motivation and Contributions}

In this study, there's an idea which could be considered as a motivation.

Question 1.1. Is it possible to use mixed versions of ideas concerning "connectedness", "neutrosophic graphs" and "neutrosophic coloring" to define some notions which are applied to neutrosophic graphs? 
It's motivation to find notions to use in any classes of neutrosophic graphs. Real-world applications about time table and scheduling are another thoughts which lead to be considered as motivation. Connections amid two items have key roles to assign colors. Thus they're used to define new ideas which conclude to the structure of coloring. The concept of having vital edge from connectedness inspires me to study the behavior of vital edge in the way that, both vital chromatic number and n-vital number are the cases of study.

The framework of this study is as follows. In the beginning, I introduced basic definitions to clarify about preliminaries. In section "Definitions and Clarification", new notion of coloring is applied to the vertices of neutrosophic graphs. Vital edge from connectedness has the key role in this way. Classes of neutrosophic graphs are studied in the terms of vital edges. In section "Applications in Time Table and Scheduling", one application is posed for neutrosophic graphs concerning time table and scheduling when the suspicions are about choosing some subjects. In section "Open Problems", some problems and questions for further studies are proposed. In section "Conclusion and Closing Remarks", gentle discussion about results and applications are featured. In section "Conclusion and Closing Remarks", a brief overview concerning advantages and limitations of this study alongside conclusions are formed.

\subsection{Preliminaries}

Definition 1.2. $G:(V, E)$ is called a crisp graph where $V$ is a set of objects and $E$ is a subset of $V \times V$ such that this subset is symmetric.

Definition 1.3. A crisp graph $G:(V, E)$ is called a neutrosophic graph $G:(\sigma, \mu)$ where $\sigma=\left(\sigma_{1}, \sigma_{2}, \sigma_{3}\right): V \rightarrow[0,1]$ and $\mu=\left(\mu_{1}, \mu_{2} . \mu_{3}\right): E \rightarrow[0,1]$ such that $\mu(x y) \leq \sigma(x) \wedge \sigma(y)$ for all $x y \in E$.

Definition 1.4. A neutrosophic graph is called neutrosophic empty if it has no edge. It's also called neutrosophic trivial. A neutrosophic graph which isn't neutrosophic empty, is called neutrosophic nontrivial.

Definition 1.5. A neutrosophic graph $G:(\sigma, \mu)$ is called a neutrosophic complete where it's complete and $\mu(x y)=\sigma(x) \wedge \sigma(y)$ for all $x y \in E$.

Definition 1.6. A neutrosophic graph $G:(\sigma, \mu)$ is called a neutrosophic strong where $\mu(x y)=\sigma(x) \wedge \sigma(y)$ for all $x y \in E$.

Definition 1.7. A path $v_{0}, v_{1}, \cdots, v_{n}$ is called neutrosophic path where $\mu\left(v_{i} v_{i+1}\right)>0, i=0,1, \cdots, n-1$. $i$-path is a path with $i$ edges, it's also called length of path.

Definition 1.8. A crisp cycle $v_{0}, v_{1}, \cdots, v_{n}, v_{0}$ is called neutrosophic cycle where there are two edges $x y$ and $u v$ such that $\mu(x y)=\mu(u v)=\bigwedge_{i=0,1, \cdots, n-1} \mu\left(v_{i} v_{i+1}\right)$.

Definition 1.9. A neutrosophic graph is called neutrosophic t-partite if $V$ is partitioned to $t$ parts, $V_{1}, V_{2}, \cdots, V_{t}$ and the edge $x y$ implies $x \in V_{i}$ and $y \in V_{j}$ where $i \neq j$. If it's neutrosophic complete, then it's denoted by $K_{\sigma_{1}, \sigma_{2}, \cdots, \sigma_{t}}$ where $\sigma_{i}$ is $\sigma$ on $V_{i}$ instead $V$ which mean $x \notin V_{i}$ induces $\sigma_{i}(x)=0$. If $t=2$, then it's called neutrosophic complete bipartite and it's denoted by $K_{\sigma_{1}, \sigma_{2}}$ especially, if $\left|V_{1}\right|=1$, then it's called neutrosophic star and it's denoted by $S_{1, \sigma_{2}}$. In this case, the vertex in $V_{1}$ is called center and if a vertex joins to all vertices of neutrosophic cycle, it's called neutrosophic wheel and it's denoted by $W_{1, \sigma_{2}}$.

Definition 1.10. Let $G:(\sigma, \mu)$ be a neutrosophic graph. For any given subset $N$ of $V$, $\Sigma_{n \in N} \sigma(n)$ is called neutrosophic cardinality of $N$ and it's denoted by $|N|_{n}$. 
Definition 1.11. Let $G:(\sigma, \mu)$ be a neutrosophic graph. Neutrosophic cardinality of $V$ is called neutrosophic order of $G$ and it's denoted by $O_{n}(G)$.

Definition 1.12. Let $G:(\sigma, \mu)$ be a neutrosophic graph. The number of vertices is denoted by $n$ and the number of edges is denoted by $m$.

Definition 1.13. Let $N=(\sigma, \mu)$ be a neutrosophic graph. It's called neutrosophic connected if for every given couple of vertices, there's at least one neutrosophic path amid them.

Definition 1.14. Let $N=(\sigma, \mu)$ be a neutrosophic graph. Suppose a path $P: v_{0}, v_{1}, \cdots, v_{n-1}, v_{n}$ from $v_{0}$ to $v_{n} . \min _{i=0,1,2, \cdots, n-1} \mu\left(v_{i} v_{i+1}\right)$ is called neutrosophic strength of $P$ and it's denoted by $\mathcal{S}_{n}(P)$.

Definition 1.15. Let $N=(\sigma, \mu)$ be a neutrosophic graph. The number of maximum edges for a vertex, amid all vertices, is denoted by $\Delta(N)$.

\section{Definitions and Clarification}

Definition 2.1. Let $N=(\sigma, \mu)$ be a neutrosophic graph. A neutrosophic edge $x y$ is called vital if deletion of $x y$ has no change on its connectedness which is a maximum strength of paths amid them.

Definition 2.2. Let $N=(\sigma, \mu)$ be a neutrosophic graph. A vertex which has common vital edge with another vertex, has assigned different color from that vertex. The number of different colors, is called vital chromatic number and its neutrosophic cardinality is called n-vital chromatic number.

Example 2.3. Assume Figure (1) with respect to first order.

(i) : Only vital edge is $n_{2} n_{3}$. Other edges aren't vital.

(ii) : The vertices $n_{2}$ and $n_{3}$ have different colors.

(iii) : The vertex $n_{1}$ could get any color.

(iv) : The vertex $n_{1}$ has no vital edge with any given vertex.

$(v)$ : The set of representatives of colors is $\left\{n_{1}, n_{2}\right\}$.

(vi) : Amid $n_{2}$ and $n_{3}, n_{2}$ has minimum value.

(vii) : Deletion of edge $n_{1} n_{2}$ has no change in the connectedness of obtained neutrosophic graph.

(viii) : The vital number is two.

$(i x):$ n-vital chromatic number is 2.57 .

\section{Basic Properties}

Proposition 3.1. Let $N=(\sigma, \mu)$ be a neutrosophic cycle. Then all edges are vital. 


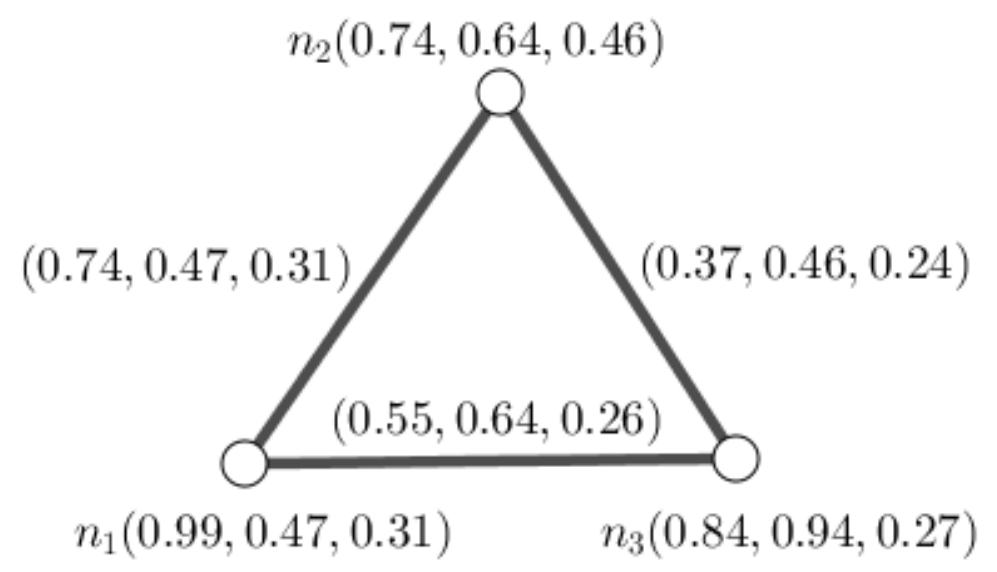

$N_{1}$

Figure 1. Neutrosophic graph $N_{1}$ is considered with respect to first order. It's complete but it isn't neutrosophic complete. It's cycle but it isn't neutrosophic cycle. It's neutrosophic 3-partite but it isn't neutrosophic complete 3-partite.

Proof. Consider $N=(\sigma, \mu)$ be a neutrosophic cycle. Hence, there are at least two edges which are weakest, it means there are $x y, u v \in E$ such that

$$
\mu(u v)=\mu(x y)=\min _{e \in E} \mu(e) .
$$

In other hand, for every given vertices $x$ and $y$, there are two paths from $x$ to $y$. So for every given path,

$$
S(P)=\min _{e \in E} \mu(e) .
$$

Thus for every $x, y \in V, x y \in E$, the value $\mu(x y)$ forms the connectedness amid $x$ to $y$. Therefore connectedness amid any given couple of vertices, doesn't change when they form an edge and they're deleted. It induces every edge is vital.

Proposition 3.2. Let $N=(\sigma, \mu)$ be a neutrosophic complete which is neither neutrosophic empty nor neutrosophic path. Then all edges are vital.

Proof. Suppose $N=(\sigma, \mu)$ is a neutrosophic complete which is neither neutrosophic empty nor neutrosophic path. If $x, y \in V$, then $x y \in E$. Thus $P: x, y$ is a path for every given couple of vertices. Hence

$$
S(P)=\mu(x y) .
$$

Therefore, connectedness $\geq \mu(x y)$. In other hands, assume $P^{\prime}: x, \cdots, y$ is an arbitrary path from $x$ to $y$. By $N=(\sigma, \mu)$ is a neutrosophic complete, $N=(\sigma, \mu)$ is a neutrosophic strong. By $N=(\sigma, \mu)$ is a neutrosophic strong,

$$
S\left(P^{\prime}\right) \leq \mu(x y) .
$$

Then connectedness $\leq S(P)$. It implies connectedness $\leq \mu(x y)$. To sum it up, connectedness $=\mu(x y)$. It induces $x y$ is vital.

Proposition 3.3. Let $N=(\sigma, \mu)$ be a neutrosophic graph which is fixed-edge and which is neither neutrosophic empty nor neutrosophic path. Then all edges are vital. 
Proof. Assume $N=(\sigma, \mu)$ is a neutrosophic graph which is fixed-edge and which is neither neutrosophic empty nor neutrosophic path. $\operatorname{By} N=(\sigma, \mu)$ is a fixed-edge,

$$
\forall e, e^{\prime} \in E, \mu(e)=\mu\left(e^{\prime}\right) .
$$

It induces for every given edge $e$ and every given paths $P, P^{\prime}$

$$
S(P)=S\left(P^{\prime}\right)=\mu(e) .
$$

It implies connectedness is fixed and it equals to $\mu(e)$ where $e \in E$. Therefore, the deletion of $e$ has no change on connectedness amid every couple of vertices. It means every edge is vital.

Proposition 3.4. Let $N=(\sigma, \mu)$ be a neutrosophic graph which is neither neutrosophic empty nor neutrosophic path. Then there's at least one vital edge.

Proof. Consider $N=(\sigma, \mu)$ is a neutrosophic graph which is neither neutrosophic empty nor neutrosophic path. Assume $N=(\sigma, \mu)$ is a neutrosophic graph which is either fixed-edge or fixed-vertex and neutrosophic strong. Hence, all edges have same value. It means

$$
\forall e, e^{\prime} \in E, \mu(e)=\mu\left(e^{\prime}\right) \text {. }
$$

It induces for every given edge $e$ and every given paths $P, P^{\prime}$

$$
S(P)=S\left(P^{\prime}\right)=\mu(e) .
$$

It implies connectedness is fixed and it equals to $\mu(e)$ where $e \in E$. Therefore, the deletion of $e$ has no change on connectedness amid every couple of vertices. It means every edge is vital. In other hand, suppose otherwise. So by $|E|>2$, there's one edge $e$ such that for every edge $e^{\prime} \neq e$,

$$
\mu(e)>\mu\left(e^{\prime}\right)
$$

Let a number $\mu\left(e^{\prime}\right)$ be

$$
\min _{e \in E} \mu(e) .
$$

Then connectedness is $\geq \mu\left(e^{\prime}\right)$. But there's a cycle which implies $|E|>3$. It induces there are at least two paths corresponded to $e^{\prime}$. By $\mu(e)>\mu\left(e^{\prime}\right)$, connectedness $\geq \mu\left(e^{\prime}\right)$. It implies corresponded connectedness to $e^{\prime}$ isn't changed when the deletion of $e^{\prime}$ is done. Thus the edge $e^{\prime} \in E$ is vital.

Proposition 3.5. Let $N=(\sigma, \mu)$ be a neutrosophic strong which is fixed-vertex and which is neither neutrosophic empty nor neutrosophic path. Then all edges are vital.

Proof. Assume $N=(\sigma, \mu)$ is a neutrosophic strong which is fixed-vertex and which is neither neutrosophic empty nor neutrosophic path. Thus by $N=(\sigma, \mu)$ is a neutrosophic fixed-vertex, for all $v, v^{\prime} \in V$,

$$
\sigma(v)=\sigma\left(v^{\prime}\right)
$$

By $N=(\sigma, \mu)$ is a neutrosophic strong, for all $e, e^{\prime} \in V$,

$$
\mu(e)=\mu\left(e^{\prime}\right)
$$

It induces for every couple of vertices which form an edge, connectedness amid them is same and equals $\mu(e)$ where $e$ is a given edge. It implies at least there are two paths with strength $\mu(e)$. Thus deletion of every edge has no change on connectedness amid its vertices. Therefore, every edge is vital. 
Proposition 3.6. Let $N=(\sigma, \mu)$ be a neutrosophic graph which is fixed-vertex and complete. Then all edges are vital.

Proof. By $N=(\sigma, \mu)$ is neutrosophic complete, $N=(\sigma, \mu)$ is neutrosophic strong. By $N=(\sigma, \mu)$ is a neutrosophic graph which is fixed-vertex, complete and applying Proposition (3.5), all edges are vital.

Proposition 3.7. Let $N=(\sigma, \mu)$ be a neutrosophic graph which is fixed-edge. Then all edges are vital.

Proof. Suppose $N=(\sigma, \mu)$ is a neutrosophic graph which is fixed-edge. Then for every edges $e$ and $e^{\prime}$,

$$
\mu(e)=\mu\left(e^{\prime}\right) .
$$

It means all paths has same strength which is the value of an edge since all edges have same values. It means the connectedness amid all given couple of vertices is the same. There are at least two paths. So deletion any edge has no change on the connectedness amid all given couple of vertices.

\section{Vital Chromatic Number}

Proposition 4.1. Let $N=(\sigma, \mu)$ be a neutrosophic graph which is neither neutrosophic empty nor neutrosophic path. Then vital chromatic number is at most $n$ and at least 1 .

Proof. These bounds are sharp and tight as they'll be shown in upcoming results. If there's no edge, then vital chromatic number is 1 but if the number of vertices are $n$ and they're connected to each other, then vital chromatic number is $n$.

\subsection{Largest Vital Chromatic Number}

Proposition 4.2. Let $N=(\sigma, \mu)$ be a neutrosophic complete which is neither neutrosophic empty nor neutrosophic path. Then vital chromatic number is $n$.

Proof. Consider $N=(\sigma, \mu)$ is a neutrosophic complete which is neither neutrosophic empty nor neutrosophic path. By Proposition (3.2), all edges are vital. By $N=(\sigma, \mu)$ isn't a neutrosophic path, there are at least two path amid two given edges. In other words, there is at least one cycle. By $N=(\sigma, \mu)$ is a neutrosophic complete, all vertices are connected to each other. It implies,

$$
\forall v, v^{\prime} \in V, v v^{\prime} \in E .
$$

It induces all vertices have different colors. The number of vertices are $n$. So vital chromatic number is $n$.

Proposition 4.3. Let $N=(\sigma, \mu)$ be a neutrosophic path. Then vital chromatic number aren't computable.

Proof. Assume $N=(\sigma, \mu)$ is a neutrosophic path. Then there's only one path amid two given vertices. So deletion of an edge makes the connectedness amid its vertices, to be incomputable.

Proposition 4.4. Let $N=(\sigma, \mu)$ be a neutrosophic star. Then vital chromatic number aren't computable.

Proof. Consider $N=(\sigma, \mu)$ is a neutrosophic star. Hence there's only one path amid two given vertices. Thus deletion of an edge makes the connectedness amid its vertices, to be incomputable.

124

125 


\subsection{Smallest Vital Chromatic Number}

Proposition 4.5. Let $N=(\sigma, \mu)$ be a neutrosophic empty. Then vital chromatic number is 1 .

Proof. Let $N=(\sigma, \mu)$ be a neutrosophic empty. Then there's no edge. It implies all vertices have same colors where the minimum number of colors are applied. Thus vital chromatic number is 1 .

Proposition 4.6. Let $N=(\sigma, \mu)$ be a neutrosophic graph which is neither neutrosophic empty nor neutrosophic path. Then vital chromatic number isn't 1.

Proof. Assume $N=(\sigma, \mu)$ is a neutrosophic graph which is neither neutrosophic empty nor neutrosophic path. By Proposition (3.4), there's at least one vital edge.

Proposition 4.7. Let $N=(\sigma, \mu)$ be a neutrosophic cycle. Then vital chromatic number is at least 2 and at most 3.

Proof. Suppose $N=(\sigma, \mu)$ is a neutrosophic cycle. There's at least amid two vertices. By Proposition (3.1), all edges are vital. So at least the colors of two vertices are different. It implies vital chromatic number is at least 2. By applying colors on vertices in alternative ways, at most two vertices have common edges with same color. Hence vital chromatic number is at most 3 .

Proposition 4.8. Let $N=(\sigma, \mu)$ be an even neutrosophic cycle. Then vital chromatic number is 2 .

Proof. Assume $N=(\sigma, \mu)$ is an even neutrosophic cycle. By Proposition (4.6), vital chromatic number is at least 2. By applying coloring on vertices in alternative ways, two vertices with common edge, has different colors. Since the cycle has even number of edges. Thus vital chromatic number is 2 .

Proposition 4.9. Let $N=(\sigma, \mu)$ be an odd neutrosophic cycle. Then vital chromatic number is 3 .

Proof. Consider $N=(\sigma, \mu)$ is an odd neutrosophic cycle. By Proposition (3.1), all edges are vital. So by using coloring in alternative way, there are two vertices which have common edge and have same color. Thus vital chromatic number is 3 .

Proposition 4.10. Let $N=(\sigma, \mu)$ be a neutrosophic bipartite which is fixed-edge and complete. Then vital chromatic number is 2.

Proof. Suppose $N=(\sigma, \mu)$ is a neutrosophic bipartite which is fixed-edge and complete. Thus strength of every path is as same as connectedness amid two vertices is. Thus all edges are vital. By $N=(\sigma, \mu)$ is complete, all vertices from one part are connected to all vertices of another part. Every part has no connection amid its vertices so all vertices from every part, have same color. There are two parts. Thus vital chromatic number is 2 .

Proposition 4.11. Let $N=(\sigma, \mu)$ be a neutrosophic bipartite which is fixed-vertex and complete. Then vital chromatic number is 2 .

Proof. By $N=(\sigma, \mu)$ is fixed-vertex and complete, $N=(\sigma, \mu)$ is fixed-edge and complete. Therefore, by Proposition (4.10), vital chromatic number is 2 .

Proposition 4.12. Let $N=(\sigma, \mu)$ be a neutrosophic $t$-partite which is fixed-edge and complete. Then vital chromatic number is $t$. 
Proof. By $N=(\sigma, \mu)$ is fixed-edge, all edges have same value. Thus all paths have same strength. So connectedness amid two given vertices are same. Therefore all edges are vital. Inside every part, there's no edge amid two vertices. It induces the vertices of every part have same color. There are $t$ parts. It implies $t$ different colors are applied. Therefore vital chromatic number is $t$.

Proposition 4.13. Let $N=(\sigma, \mu)$ be a neutrosophic $t$-partite which is fixed-vertex and complete. Then vital chromatic number is $t$.

Proof. It's fixed-vertex and complete. So It's fixed-edge and complete. By Proposition (4.12), vital chromatic number is $t$.

Proposition 4.14. Let $N=(\sigma, \mu)$ be a neutrosophic wheel which is fixed-vertex and neutrosophic strong. Then vital chromatic number is 3 or 4.

Proof. Consider $N=(\sigma, \mu)$ is a neutrosophic wheel which is fixed-vertex and neutrosophic strong. By it's fixed-vertex and neutrosophic strong, it's fixed-edge. Every edges have same value. So strength of paths and connectedness are same and equal to each other. Thus all edges are vital. Then the center has one color and since it's connected to all other vertices, the color of center is unique. Therefore, vital chromatic number is at least 2. Non-center vertices form a path which are colored by two colors when applying colors are in alternative ways. Thus vital chromatic number is 3 if the non-center vertices form even color and vital chromatic number is 4 if the non-center vertices form odd color.

Proposition 4.15. Let $N=(\sigma, \mu)$ be a neutrosophic wheel which is fixed-edge and neutrosophic strong. Then vital chromatic number is 3 or 4.

Proof. Consider $N=(\sigma, \mu)$ is a neutrosophic wheel which is fixed-vertex and neutrosophic strong. It's fixed-edge. Every edges have same value. So strength of paths and connectedness are same and equal to each other. Thus all edges are vital. Then the center has one color and since it's connected to all other vertices, the color of center is unique. Therefore, vital chromatic number is at least 2. Non-center vertices form a path which are colored by two colors when applying colors are in alternative ways. Thus vital chromatic number is 3 if the non-center vertices form even color and vital chromatic number is 4 if the non-center vertices form odd color.

\section{5 n-Vital Chromatic Number}

Proposition 5.1. Let $N=(\sigma, \mu)$ be a neutrosophic graph which is neither neutrosophic empty nor neutrosophic path. Then n-vital chromatic number is at most order of $N$ which is neutrosophic cardinality of $V$.

Proof. Assume $N=(\sigma, \mu)$ is a neutrosophic graph which is neither neutrosophic empty nor neutrosophic path. If all edges are vital and all vertices are connected to each other, then vital chromatic number is $n$. Thus n-vital chromatic number is at most order of $N$ which is neutrosophic cardinality of $V$.

\subsection{Largest n-Vital Chromatic Number}

Proposition 5.2. Let $N=(\sigma, \mu)$ be a neutrosophic complete which is neither neutrosophic empty nor neutrosophic path. Then n-vital chromatic number is order of $N$ which is neutrosophic cardinality of $V$. 
Proof. Suppose $N=(\sigma, \mu)$ is a neutrosophic complete which is neither neutrosophic empty nor neutrosophic path. By it's complete, then all vertices are connected to each other and all edges are vital. Thus $n$ colors are used. It means n-vital chromatic number is order of $N$ which is neutrosophic cardinality of $V$.

Proposition 5.3. Let $N=(\sigma, \mu)$ be a neutrosophic path. Then n-vital chromatic number aren't computable.

Proof. Deletion of one edge, make $N=(\sigma, \mu)$ be in the situation where n-vital chromatic number aren't computable. Since there's need to have at least two paths to compute n-vital chromatic number. In other words, this notion is computable in neutrosophic graph which has at least one cycle.

Proposition 5.4. Let $N=(\sigma, \mu)$ be a neutrosophic star. Then n-vital chromatic number aren't computable.

Proof. Assume $N=(\sigma, \mu)$ is a neutrosophic star. Then there's only one path amid two given vertices. Deletion one edge causes the connectedness to be incomputable. Thus n-vital chromatic number aren't computable.

\subsection{Smallest n-Vital Chromatic Number}

Proposition 5.5. Let $N=(\sigma, \mu)$ be a neutrosophic empty. Then n-vital chromatic number is

$$
\min _{x \in V} \sigma(x)
$$

Proof. Suppose $N=(\sigma, \mu)$ is a neutrosophic empty. Then there's no edge. It induces there's no vital edge. So all vertices are colored by one color. Hence all vertices have same color. It means the number of color is one. It induces the cardinality of set includes the representative of color is one. To find the representative of color, we have 1 choice from $n$ options. Thus n-vital chromatic number is

$$
\min _{x \in V} \sigma(x)
$$

Proposition 5.6. Let $N=(\sigma, \mu)$ be a neutrosophic graph which is neither neutrosophic empty nor neutrosophic path. Then n-vital chromatic number isn't

$$
\min _{x \in V} \sigma(x)
$$

Proof. Consider $N=(\sigma, \mu)$ is a neutrosophic graph which is neither neutrosophic empty nor neutrosophic path. Then there's at least one edge. By Proposition (3.4), there's at least one vital edge. It induces the number of color is at least two. Therefore, the cardinality of set of representative is at least two. It implies n-vital chromatic number isn't

$$
\min _{x \in V} \sigma(x)
$$

Proposition 5.7. Let $N=(\sigma, \mu)$ be a neutrosophic cycle. Then n-vital chromatic number is at least

$$
\min _{x, y \in V, x y \in E} \sigma(x)+\sigma(y) .
$$

And at most

$$
\min _{x, y, z \in V, x y, y z, x z \in E} \sigma(x)+\sigma(y)+\sigma(z) .
$$


Proof. Suppose $N=(\sigma, \mu)$ is a neutrosophic cycle. By using alternative coloring of vertices, two or three numbers of colors are used. So the cardinality of set of representative is two or three. There are only these possibilities. Therefore n-vital chromatic number is at least

$$
\min _{x, y \in V, x y \in E} \sigma(x)+\sigma(y) .
$$

And at most

$$
\min _{x, y, z \in V, x y, y z, x z \in E} \sigma(x)+\sigma(y)+\sigma(z) .
$$

Proposition 5.8. Let $N=(\sigma, \mu)$ be an even neutrosophic cycle. Then n-vital chromatic number is

$$
\min _{x, y \in V, x y \in E} \sigma(x)+\sigma(y) .
$$

Proof. Assume $N=(\sigma, \mu)$ is an even neutrosophic cycle. If colors are applied on vertices in alternative ways which cause two vertices with a common edge, have different colors, then by it's even neutrosophic cycle, the representatives of colors are two. Since there are even edges which by Proposition (3.1), all are vital. It induces the cardinality of set of representatives is two. Thus n-vital chromatic number is n-vital chromatic number is

$$
\min _{x, y \in V, x y \in E} \sigma(x)+\sigma(y) .
$$

Proposition 5.9. Let $N=(\sigma, \mu)$ be an odd neutrosophic cycle. Then n-vital chromatic number is

$$
\min _{x, y, z \in V, x y \in E} \sigma(x)+\sigma(y)+\sigma(z) .
$$

Proof. Consider $N=(\sigma, \mu)$ is an odd neutrosophic cycle. Then number of edges are odd. By Proposition (3.1), all edges are vital. Using different colors on the vertices which have common edges, implies usage of three colors. Hence the set of representatives has the cardinality three. To choose, the representatives, in every color, minimum value of vertices, introduces the representative of specific color. Then n-vital chromatic number is

$$
\min _{x, y, z \in V, x y \in E} \sigma(x)+\sigma(y)+\sigma(z) .
$$

Proposition 5.10. Let $N=(\sigma, \mu)$ be neutrosophic bipartite which is fixed-edge and complete. Then n-vital chromatic number is

$$
\min _{x, y \in V, x y \in E} \sigma(x)+\sigma(y) .
$$

Proof. Assume $N=(\sigma, \mu)$ is neutrosophic bipartite which is fixed-edge and complete. It's fixed-edge so all edges have same value and as its consequences, all paths have same strength and all connectedness are same. Hence all edges are vital. By it's complete, all vertices from one part are connected to all vertices from another part. By it's bipartite, there are two colors to use on vertices such that every part has same color. So the set of representatives has the cardinality two which implies n-vital chromatic number is

$$
\min _{x, y \in V, x y \in E} \sigma(x)+\sigma(y) .
$$


Proposition 5.11. Let $N=(\sigma, \mu)$ be neutrosophic bipartite which is fixed-vertex and complete. Then n-vital chromatic number is

$$
\min _{x, y \in V, x y \in E} \sigma(x)+\sigma(y) .
$$

Proof. Assume $N=(\sigma, \mu)$ is neutrosophic bipartite which is fixed-vertex and complete. By it's fixed-vertex and complete, it's fixed-edge and complete. By Proposition (5.10), n-vital chromatic number is

$$
\min _{x, y \in V, x y \in E} \sigma(x)+\sigma(y)
$$

Proposition 5.12. Let $N=(\sigma, \mu)$ be neutrosophic $t$-partite which is fixed-edge and complete. Then n-vital chromatic number is

$$
\min _{x_{1}, x_{2}, \cdots, x_{t} \in V, x_{i} x_{j} \in E} \sigma\left(x_{1}\right)+\sigma\left(x_{2}\right)+\cdots+\sigma\left(x_{t}\right) .
$$

Proof. Assume $N=(\sigma, \mu)$ is neutrosophic $t$-partite which is fixed-edge and complete. All parts have same color on their vertices. By it's fixed-edge and applying Proposition (3.7), all edges are vital. Thus minimum number of colors is $t$. And the set of representatives has the cardinality $t$. It means $n$-vital chromatic number is

$$
\min _{x_{1}, x_{2}, \cdots, x_{t} \in V, x_{i} x_{j} \in E} \sigma\left(x_{1}\right)+\sigma\left(x_{2}\right)+\cdots+\sigma\left(x_{t}\right) .
$$

Proposition 5.13. Let $N=(\sigma, \mu)$ be neutrosophic $t$-partite which is fixed-vertex and complete. Then n-vital chromatic number is

$$
\min _{x_{1}, x_{2}, \cdots, x_{t} \in V, x_{i} x_{j} \in E} \sigma\left(x_{1}\right)+\sigma\left(x_{2}\right)+\cdots+\sigma\left(x_{t}\right) .
$$

Proof. Assume $N=(\sigma, \mu)$ is neutrosophic $t$-partite which is fixed-vertex and complete. Then by it's fixed-vertex and complete, it's it's fixed-edge and complete. By Proposition (5.12), n-vital chromatic number is

$$
\min _{x_{1}, x_{2}, \cdots, x_{t} \in V, x_{i} x_{j} \in E} \sigma\left(x_{1}\right)+\sigma\left(x_{2}\right)+\cdots+\sigma\left(x_{t}\right) .
$$

Proposition 5.14. Let $N=(\sigma, \mu)$ be neutrosophic wheel which is fixed-vertex and neutrosophic strong. Then n-vital chromatic number is

$$
\min _{y, z \in V, y z \in E} \sigma(c)+\sigma(y)+\sigma(z) .
$$

Or

$$
\min _{y, z \in V, y z, z t \in E} \sigma(c)+\sigma(y)+\sigma(z)+\sigma(t) .
$$

Proof. Consider $N=(\sigma, \mu)$ is neutrosophic wheel which is fixed-vertex and neutrosophic strong. By fixed-vertex and neutrosophic strong, it's fixed-edge. By it's fixed-edge and applying Proposition (3.7), all edges are vital. Center is connected to non-center vertices. So center uses unique color. Non-center vertices form a cycle. If the cycle is even, then $\mathrm{n}$-vital chromatic number is

$$
\min _{y, z \in V, y z \in E} \sigma(c)+\sigma(y)+\sigma(z) .
$$


If it's odd, then n-vital chromatic number is

$$
\min _{y, z \in V, y z \in E} \sigma(c)+\sigma(y)+\sigma(z)
$$

Or

$$
\min _{y, z \in V, y z, z t \in E} \sigma(c)+\sigma(y)+\sigma(z)+\sigma(t)
$$

Proposition 5.15. Let $N=(\sigma, \mu)$ be neutrosophic wheel which is fixed-edge and neutrosophic strong. Then n-vital chromatic number is

$$
\min _{y, z \in V, y z \in E} \sigma(c)+\sigma(y)+\sigma(z) .
$$

Proof. Assume $N=(\sigma, \mu)$ is neutrosophic wheel which is fixed-edge and neutrosophic strong. By it's fixed-edge and neutrosophic strong, it's fixed-vertex and neutrosophic strong. By Proposition (5.14),

$$
\min _{y, z \in V, y z \in E} \sigma(c)+\sigma(y)+\sigma(z)
$$

$\mathrm{Or}$

$$
\min _{y, z \in V, y z, z t \in E} \sigma(c)+\sigma(y)+\sigma(z)+\sigma(t)
$$

The relation amid neutrosophic chromatic number and main parameters of neutrosophic graphs is computed.

Proposition 5.16. Let $N=(\sigma, \mu)$ be a neutrosophic strong. Then vital chromatic number is at most $\Delta+1$ and at least 2 .

Proof. Neutrosophic strong is neutrosophic nontrivial. So it isn't neutrosophic empty which induces there's no edge. It implies chromatic number is two. Since chromatic number is one if and only if $N=(\sigma, \mu)$ is neutrosophic empty if and only if $N=(\sigma, \mu)$ is neutrosophic trivial. A vertex with degree $\Delta$, has $\Delta$ vertices which have common edges with them. If these vertices have no edge amid each other, then chromatic number is two especially, neutrosophic star. If not, then in the case, all vertices have edge amid each other, chromatic number is $\Delta+1$, especially, neutrosophic complete.

Proposition 5.17. Let $N=(\sigma, \mu)$ be a neutrosophic $r$-regular. Then vital chromatic number is at most $r+1$.

Proof. $N=(\sigma, \mu)$ is a neutrosophic $r$-regular. So any of vertex has $r$ vertices which have common edge with it. If these vertices have no common edge with each other, for instance neutrosophic star, chromatic number is two. But since the vertices have common edge with each other, chromatic number is $r+1$, for instance, neutrosophic complete.

\section{Applications in Time Table and Scheduling}

Designing the programs to achieve some goals is general approach to apply on some issues to function properly. Separation has key role in the context of this style. Separating the duration of work which are consecutive, is the matter and it has important to avoid mixing up. 
Step 1. (Definition) Time table is an approach to get some attributes to do the work fast and proper. The style of scheduling implies special attention to the tasks which are consecutive.

Step 2. (Issue) Scheduling of program has faced with difficulties to differ amid consecutive section. Beyond that, sometimes sections are not the same.

Step 3. (Model) As Figure (2), the situation is designed as a model. The model uses data to assign every section and to assign to relation amid section, three numbers belong unit interval to state indeterminacy, possibilities and determinacy. There's one restriction in that, the numbers amid two sections are at least the number of the relation amid them. Table (1), clarifies about the assigned numbers to these situation.
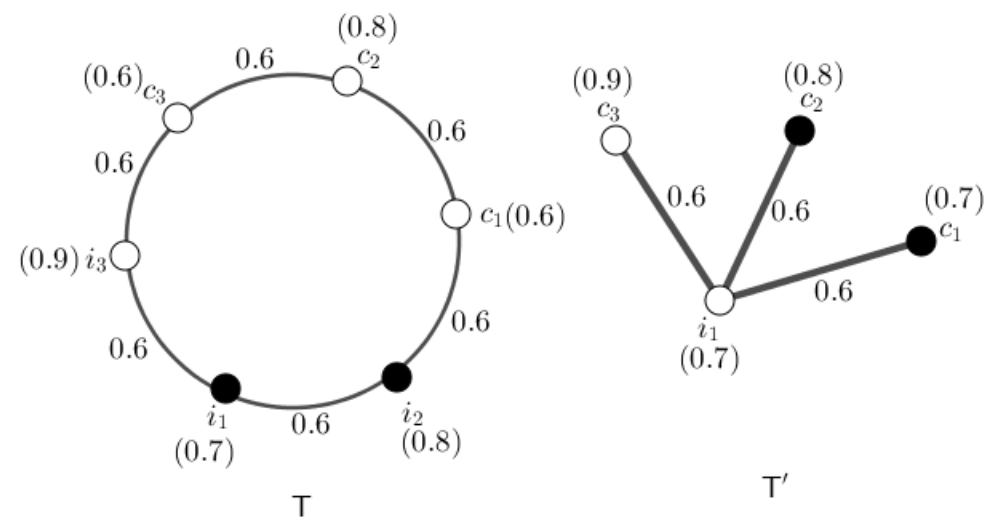

$\mathrm{T}$

$\mathrm{T}^{\prime}$

Figure 2. Black vertices are suspicions about choosing them.

Table 1. Scheduling concerns its Subjects and its Connections as a Neutrosophic Graph in a Model.

\begin{tabular}{c|lcccccccc}
\hline Sections of $T$ & $s_{1}$ & $s_{2}$ & $s_{3}$ & $s_{4}$ & $s_{5}$ & $s_{6}$ & $s_{7}$ & $s_{8}$ & $s_{9}, s_{10}$ \\
\hline Values & 0.1 & 0.8 & 0.7 & 0.8 & 0.1 & 0.3 & 0.6 & 0.5 & 0.2 \\
Connections of $T$ & $s_{1} s_{2}$ & $s_{2} s_{3}$ & $s_{3} s_{4}$ & $s_{4} s_{5}$ & $s_{5} s_{6}$ & $s_{6} s_{7}$ & $s_{7} s_{8}$ & $s_{8} s_{9}$ & $s_{9} s_{10}$ \\
Values & 0.1 & 0.6 & 0.4 & 0.1 & 0.1 & 0.2 & 0.4 & 0.2 & 0.1 \\
\hline
\end{tabular}

Step 4. (Solution) As Figure (2) shows, neutrosophic model, proposes to use vital chromatic number which is incomputable in the case which is titled $T^{\prime}$. In this case, $i_{1}$ and $c_{1}$ aren't representative of these two colors and n-vital chromatic number is incomputable. The set $\left\{i_{1}, c_{1}\right\}$ doesn't contain representatives of colors which pose vital chromatic number and n-vital chromatic number. Thus the decision amid choosing the subject $c_{1}$ an $c_{2}$ isn't concluded to choose $c_{1}$. To get brief overview, neutrosophic model uses one number for every array so 0.9 means $(0.9,0.9,0.9)$. In Figure $(2)$, the neutrosophic model $T$ introduces the common situation. The representatives of colors are $i_{2}$ and $c_{1}$. Thus vital chromatic number is two and n-vital chromatic number is 1.4. Thus suspicion about choosing $i_{1}$ and $i_{2}$ is determined to be $i_{2}$. The sets of representative for colors are $\left\{i_{2}, c_{1}\right\}$.

\section{Open Problems}

The two notions of coloring of vertices concerning vital chromatic number and n-vital chromatic number are defined on neutrosophic graphs when connectedness and as its 
consequences, vital edges have key role to have these notions. Thus

Question 7.1. Is it possible to use other types edges via connectedness to define vital chromatic number and n-vital chromatic number?

Question 7.2. Are existed some connections amid the coloring from connectedness inside this concept and external connections with other types of coloring from other notions?

Question 7.3. Is it possible to construct some classes neutrosophic graphs which have "nice" behavior?

Question 7.4. Which applications do make an independent study to apply vital chromatic number and n-vital chromatic number?

Problem 7.5. Which parameters are related to this parameter?

Problem 7.6. Which approaches do work to construct applications to create independent study?

Problem 7.7. Which approaches do work to construct definitions which use all three arrays and the relations amid them instead of one array of three arrays to create independent study?

\section{Conclusion and Closing Remarks}

This study uses mixed combinations of vital chromatic number and n-vital chromatic number to study on neutrosophic graphs. The connections of vertices which are clarified by vital edges from connectedness, differ them from each other and and put them in different categories to represent one representative for each color. Further studies could be about changes in the settings to compare this notion amid different settings of graph theory. One way is finding some relations amid array of vertices to make sensible definitions. In Table (2), some limitations and advantages of this study is pointed out.

Table 2. A Brief Overview about Advantages and Limitations of this study

\begin{tabular}{|c|c|}
\hline Advantages & Limitations \\
\hline $\begin{array}{c}\text { 1. Using connectedness for vital edges } \\
\text { 2. Using neutrosophic cardinality }\end{array}$ & 1. Acyclic neutrosophic graphs \\
3. Using cardinality & 2. Connections with parameters \\
4. Characterizing smallest number & \\
5. Characterizing biggest number & 3. Star and path \\
\hline
\end{tabular}

\section{References}

1. M. Akram, and G. Shahzadi, Operations on Single-Valued Neutrosophic Graphs, Journal of uncertain systems 11 (1) (2017) 1-26.

2. K. Atanassov, Intuitionistic fuzzy sets, Fuzzy Sets Syst. 20 (1986) 87-96. 
3. S. Broumi, M. Talea, A. Bakali and F. Smarandache, Single-valued neutrosophic graphs, Journal of New Theory 10 (2016) 86-101.

4. N. Shah, and A. Hussain, Neutrosophic soft graphs, Neutrosophic Set and Systems 11 (2016) 31-44.

5. Henry Garrett, Big Sets Of Vertices, Preprints 2021, 2021060189 (doi: 10.20944/preprints202106.0189.v1).

6. Henry Garrett, Locating And Location Number, Preprints 2021, 2021060206 (doi: 10.20944/preprints202106.0206.v1).

7. Henry Garrett, Metric Dimensions Of Graphs, Preprints 2021, 2021060392 (doi: 10.20944/preprints202106.0392.v1).

8. Henry Garrett, New Graph Of Graph, Preprints 2021, 2021060323 (doi: 10.20944/preprints202106.0323.v1).

9. Henry Garrett, Numbers Based On Edges, Preprints 2021, 2021060315 (doi: 10.20944/preprints202106.0315.v1).

10. Henry Garrett, Matroid And Its Outlines, Preprints 2021, 2021060146 (doi: 10.20944/preprints202106.0146.v1).

11. Henry Garrett, Matroid And Its Relations, Preprints 2021, 2021060080 (doi: 10.20944/preprints202106.0080.v1).

12. A. Shannon and K.T. Atanassov, A first step to a theory of the intuitionistic fuzzy graphs, Proceeding of FUBEST (Lakov, D., Ed.) Sofia (1994) 59-61.

13. F. Smarandache, A Unifying field in logics neutrosophy: Neutrosophic probability, set and logic, Rehoboth: American Research Press (1998).

14. H. Wang, F. Smarandache, Y. Zhang, and R. Sunderraman, Single-valued neutrosophic sets, Multispace and Multistructure 4 (2010) 410-413.

15. L. A. Zadeh, Fuzzy sets, Information and Control 8 (1965) 338-353. 